\title{
STUDY ON MOMENT-ROTATION RELATIONSHIP OF STEEL SLEEVE BEAM-COLUMN JOINT WITH INTERFERENCE FIT
}

\author{
Wen-Tao Qiao ${ }^{1,2,{ }^{*}}$, Ren-Jie Zhu ${ }^{1}$, Dong Wang ${ }^{3}$, Jian $\mathrm{Li}^{1,4}$ and Jia-Wei Yuan ${ }^{1}$ \\ ${ }^{1}$ School of Civil Engineering, Shi Jiazhuang Tiedao University, Shi Jiazhuang, China \\ ${ }^{2}$ Cooperative Innovation Center of Disaster Prevention and Mitigation for Large Infrastructure in Hebei Province (Shi Jiazhuang Tiedao University), Shi Jiazhuang, China \\ ${ }^{3}$ TRC Companies, Baton Rouge, United States of America \\ ${ }^{4}$ School of Civil Engineering, Southwest Jiaotong University, Chengdu, China \\ *(Corresponding author: E-mail: tottyer@126.com)
}

\section{A B S T R A C T}

The steel sleeve beam-column joint with interference fit (SSBCJ-IF) is a new steel structure beam-column joint based on interference fit in mechanical engineering. The refined finite element analysis (FEA) model is proposed by comparing analysis results with those of similarity test and theoretical calculations. The refined FEA model is used to perform variable parametric analyses on the moment-rotation relationship of SSBCJ-IF, including loading, material and geometry properties. Results show that the strength of concrete has little influence on both bearing capacity and initial stiffness of the joint, the strength of steel has obvious influence on flexural capacity of the joint but does not affect initial stiffness, both magnitude of interference and ratio of beam-column stiffness have some influence on the initial stiffness, the ratio of beam-column strength has some influence on both initial stiffness and bearing capacity, both initial stiffness and bearing capacity of the joint decrease significantly when the axial compression ratio exceeds 0.7 . Finally, a simplified calculation method of the moment-rotation curve is proposed, and results calculated by this simplified method are in good agreement with those from the refined FEA.
ART I CLE H IS T O RY

$\begin{array}{ll}\text { Received: } & 23 \text { July } 2020 \\ \text { Revised: } & 13 \text { August } 2020 \\ \text { Accepted: } & 10 \text { September } 2020\end{array}$

\section{K E Y W O R D S}

Steel sleeve beam-column joint; Interference fit;

Finite element analysis;

Parametric analysis;

Moment-rotation curve:

\section{Introduction}

Concrete-filled steel tubes (CFST) have become increasingly popular in construction and infrastructure projects because of the advantages of fast erection, high ductility of steel and high compressive strength of concrete. CFST has these advantages because the steel tube provides confinement for concrete which increase concrete stiffness and strength, while local and global buckling of the steel tube are inhibited by concrete.

Over the past several decades, researchers and structural engineers have proposed a wide variety of joints, including the joint with the external/internal diaphragm[1], joint with through diaphragm[2], joint with through web/flange/beam [3-5], joint with external stiffeners [6], joint using bolted connections[7-10] and so on.

The aforementioned joints need to be welded or bolted on site, and some of them require cutting on steel column. Based on the interference fit in mechanical engineering, a new joint design is proposed in this paper, namely steel sleeve beam-column joint with interference fit (SSBCJ-IF), to reduce construction difficulties. The new joint is composed of the sleeve and the cantilever short beam as illustrated in Fig.1. During construction, a connection between beams and columns can be achieved by pressing the sleeve onto the column, thus both on-site welding and column cutting are avoided.

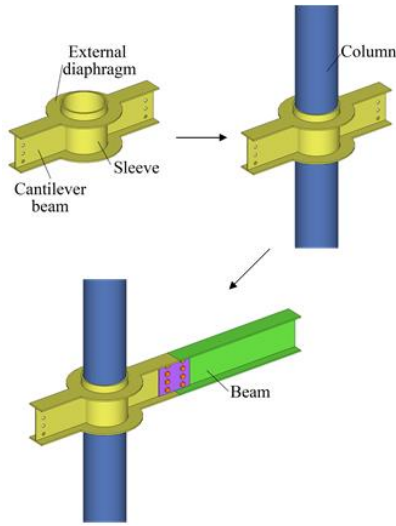

Fig. 1 Assembly process of the steel sleeve joint

In design practice, the connection between the beam and column is usually simplified as either rigid or pin connection. In reality, the ideal rigid or pin connection does not exist, and most of joints are semi-rigid connections. To clarify the influence of the beam-column angle change on the structure behavior and to represent the nonlinear behavior of the joint, the moment-rotation $(M-\theta)$ curve is usually used to describe the mechanical properties of the connection between beams and columns. Many calculation models for moment-rotation curve were proposed. Early studies use linear model to represent the connection characteristics for the whole loading process [11], but the linear model is only accurate for the elastic behavior of the connection. Frye et al [12] proposed a polynomial model which can be easily used in structural analysis. The disadvantage of this model is that the polynomial has negative slope in some ranges which results in negative stiffness. B-spline model [13] can avoid the negative stiffness issue and give results in good agreement with those from test, however, more test data are needed to determine the B-spline model. Kishi and Chen [14] proposed a three-parameter power function model which requires much less data for function fitting than the data needed for the B-spline model. Also, the three-parameter power function model is applicable to the situation in which the bending moment-angle curve is flat at late levels of loading. Yee and Melchers [15] proposed the four-parameter exponential model. Although the moment-rotation curve given by the mathematical model is not as accurate as that obtained from experimental study or finite element analysis, the curves from the mathematical models are widely used due to the simplicity and convenience [16].

In this paper, a refined FEA model of SSBCJ-IF is established. The FEA results are compared with the results from test and theoretical calculation to verify the reliability of the FEA model. Then, variable parametric analyses are performed, with the parameters being categorized into three groups including loading, material and geometry properties. Finally, a simplified calculation method of moment-rotation relationship of SSBCJ-IF is proposed.

\section{FEA modeling}

As seen in Fig.2(a) is model dimension. The section size of steel column is $273 \mathrm{~mm} \times 7 \mathrm{~mm}$ (diameter $\times$ wall thickness) filled with $\mathrm{C} 40$ concrete.The beam section size height, width, web thickness, flange thickness is $250 \mathrm{~mm}$, $125 \mathrm{~mm}, 6 \mathrm{~mm}, 9 \mathrm{~mm}$ respectively. Fig.2(b) show sleeve joint details. The thickness of the diaphragm is the same as that of the beam flange. There are pinned about top and bottom of the column. The axial compressive ratio is 0.3 . A pair of antisymmetric loads are applied at the beam ends. 


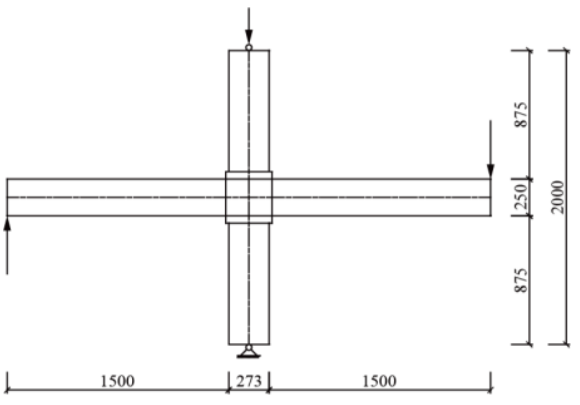

(a) Model dimension

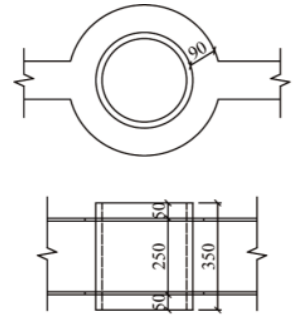

$\lcm{272.5 \quad\lfloor 16}$

(b) Steeve connection details

Fig. 2 Details of models (mm)

\subsection{Material properties}

In the model, Chinese grade Q235 was used for steel. The elastic modulus of steel $E s$ is $206 \times 10^{3} \mathrm{~N} / \mathrm{mm}^{2}$ while Poisson ratio as 0.3 [17]. The constitutive model of steel suggested in reference [18] is adopted. The stress-strain curve is divided into four segments which are elastic segment, yield segment, strain-hardening segment and secondary yield segment. C40 concrete is used in the models. Concrete Young's modulus and Poisson ratio adopt corresponding values in [19]. The damaged plasticity of concrete (CDP) model in ABAQUS is used to simulate the mechanical properties of concrete. The stress-strain curve of concrete is calculated with the method introduced in reference [20]. The plastic-damage factor of concrete is determined with the method in reference.

\subsection{Element type and meshing}

The incompatible mode eight node brick element (C3D8I) is used for the steel column, concrete and steel sleeve. Four-node quadrilateral stress/displacement shell element (S4R) is used for the diaphragm and steel beams. To improve accuracy of the model, the mesh densities of the steel sleeve and steel column are unified to make the nodes at contact surface have one-to-one correspondence. Using the symmetry of the structure, a half model of the joint is created. Finer mesh is used for the core region of the joint and less fine mesh is used for other regions, so that both calculation accuracy and efficiency are considered.

\subsection{Contact modeling and boundary condition}

The "hard contact" is used to simulate the interference fit between the sleeve and the column [21]. The tangential direction is friction. Assume the friction conforms to the Coulomb friction criterion and the friction coefficient $\mu$ is 0.1 . The "hard contact" is also used to simulate the interface between the steel tube and core concrete, while in the tangential direction the friction is considered with the Coulomb friction criterion and the friction coefficient $\mu$ is taken as $0.6[20]$.

The column are pinned on top and bottom. while axial force is applied in y direction to simulate the axial pressure in the column. The forced displacement in y direction is applied to the reference points of the beam ends. The beams are laterally braced to avoid lateral instability of the beams. Since only half of the joint is created using symmetry of the structure, the symmetric boundary conditions are applied on the plane of symmetry.

The Newton-Raphson nonlinear solution method in ABAQUS/Standard is used. The automatic control for load increment in ABAQUS/Standard is selected.

\section{Reliability of FEA modeling}

To verify the reliability of FEA modeling of SSBCJ-IF, the following methods are used.

(1) Compared with the experimental study on similar beam-column joints to verify the simulation of contact connection between the steel sleeve and steel column.

(2) Compared with the experimental study on CFST columns to verify the simulation of contact connection between the steel column and concrete core.

(3) For interference fit, if the steel sleeve and steel column are in the elastic range, the connection structure behavior can be solved as an elastic plane-strain problem. The results from this solution are used to verify the assumption of nonlinear contact between steel sleeve and steel column in FEA model.

\subsection{Beam-column joint}

The separated sleeve joint proposed in reference [22] is similar to SSBCJ-IF. For both types of joints, the sleeve connectes the steel column and the steel beam. But the connection mechanisms of these two joints are different. For separated sleeve joint, the steel beam flange is welded to the sleeve and the beam web is welded directly to the steel column. In this paper, the specimen WTJ-2 from reference [22] is selected to verify the FEA modeling. The steel column section diameter, wall thickness is $219 \mathrm{~mm}, 10 \mathrm{~mm}$ respectively. The beam section width, web thickness and flange thickness is $200 \mathrm{~mm}$, $100 \mathrm{~mm}, 5.5 \mathrm{~mm}$ and $8 \mathrm{~mm}$ respectively. The joint size is as shown in Fig. 3 .

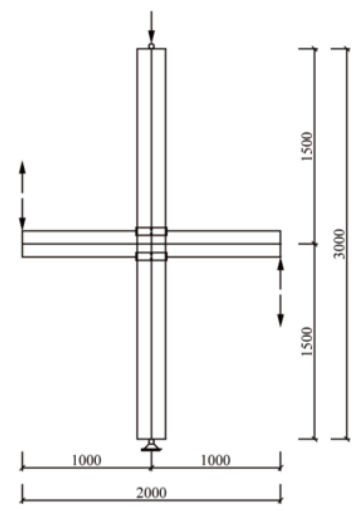

(a) Front elevation
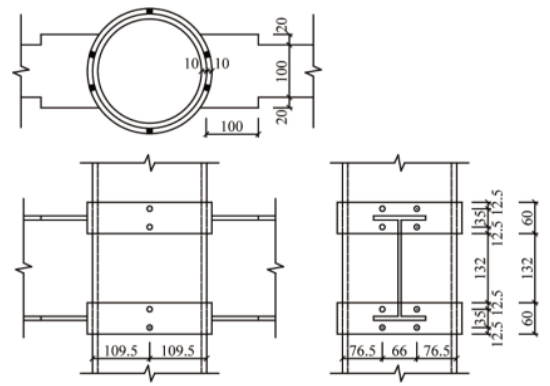

(b) Details

Fig. 3 Dimension of the specimen WTJ-2 (mm)

The FEA model is established in ABAQUS according to the settings described in Section 2. The boundary conditions are the same as those in experiments. The loading process in experiment is also simulated in FEA model. The Mises stress contour of the joint at the ultimate load state is shown in Fig.4. It is seen that the results from test and the finite element mode in Fig.5 are in good agreement,it shows that indicating that the finite element model established by the above method is reliable. 


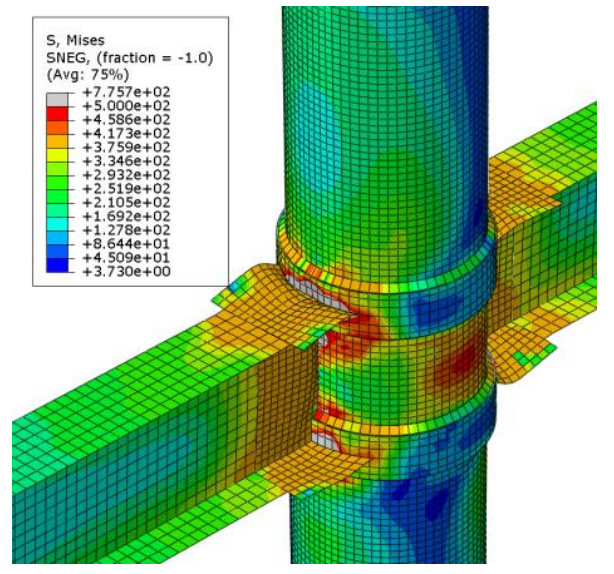

Fig. 4 WTJ-2 Mises stress contour

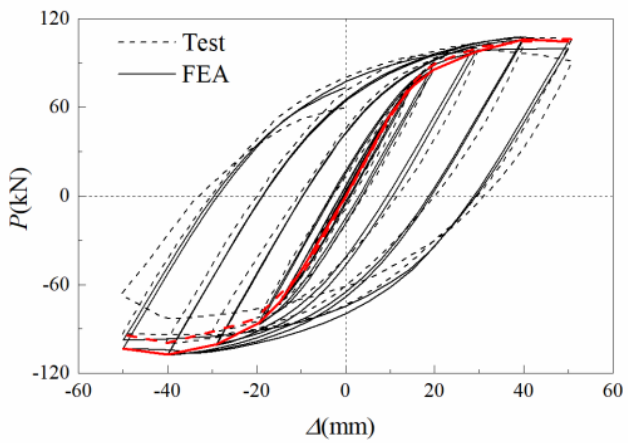

Fig. 5 Comparison of hysteresis curves between FEA calculation and test results for

specimen WTJ-2

\subsection{CFST Column}

A series of experimental studies on CFST column under reversed load were performed in reference [22]. The specimen SC2-3 from reference [23] is selected in this paper to validate the FEA model. The column section is 114 $\mathrm{mm} \times 3 \mathrm{~mm}$ and the column length is $1500 \mathrm{~mm}$. The steel tube is filled with concrete. Both ends of the CFST column are pinned. At the end of the column, the axial force is applied. The reversed load is applied in the direction perpendicular to the column at midspan.

The FEA model is established in ABAQUS according to the settings in Section 2. The boundary conditions are the same as those in experiments. Fig.6 shows the failure model. The comparison of results from FEA and experiment study is shown in Fig.7, which for hysteresis curves the stiffness from FEA at late stage of unloading and reverse loading stage are slightly greater than those from test. The skeleton curves from FEA and test match well at loading stage. The skeleton curve from FEA at reverse loading stage is slightly smaller than that from test. In general, the results of FEA and experiment are in fairly good agreement.

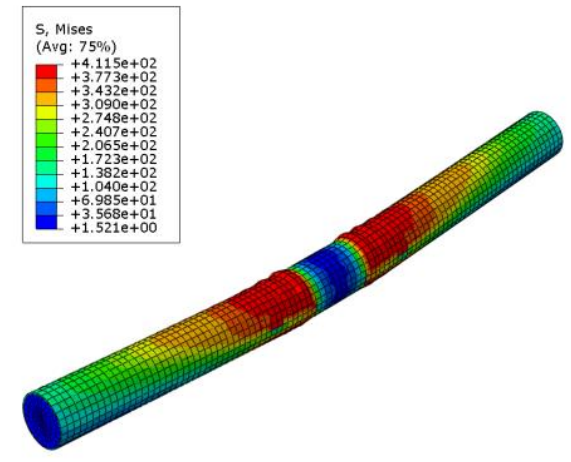

Fig.6. SC2-3 Mises stress contour plot.

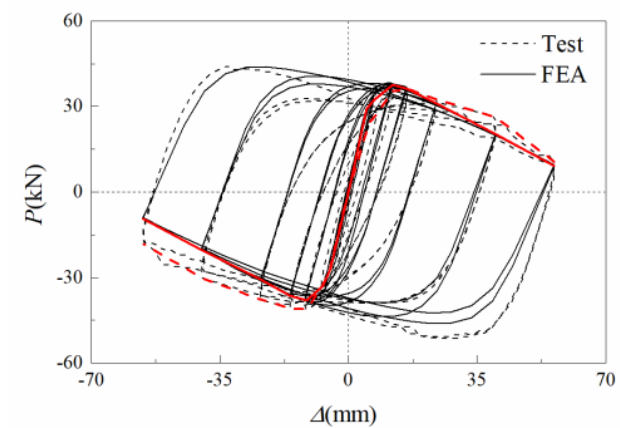

Fig. 7 Comparison of hysteresis curves from FEA and test for specimen SC2-3

\subsection{Interference Fit}

The magnitude of interference is the difference between the outer diameter of the steel column and the inner diameter of the sleeve. The mechanism of the interference fit is that each part is deformed to satisfy the compatibility of deformation. Thus, the contact pressure is generated. The steel sleeve and column can be analyzed as hollow thick-walled cylinders. If the interference fit is made before the concrete pouring and the sleeve and column do not reach plasticity stage during the interference fit, the interference fit connection structural behavior can be approximately simplified to axisymmetric plane-strain problem in elastic range.

The model described in Section 2 is selected for interference fit FEA simulation verification. The interference is $0.5 \mathrm{~mm}$.

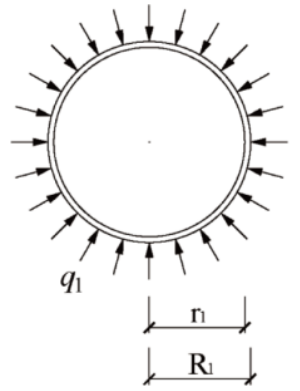

(a) Steel columı

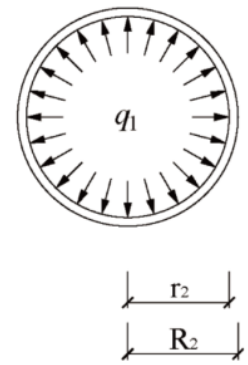

(b) Steel sleeve
Fig. 8 Stress of each part after interference fit

As shown in Fig.8, assume that the pressure between steel column and sleeve is $q_{1}$.

For steel column, the stress field is:

$$
\sigma_{\rho}=-\frac{1-\frac{r_{1}^{2}}{\rho^{2}}}{1-\frac{r_{1}^{2}}{R_{1}^{2}}} q_{1} \quad \sigma_{\varphi}=-\frac{1+\frac{r_{1}^{2}}{\rho^{2}}}{1-\frac{r_{1}^{2}}{R_{1}^{2}}} q_{1}
$$

Radial displacement is:

$u_{\rho}=\frac{1+\mu}{E}\left[-\frac{r_{1}^{2} R_{1}^{2} q_{1}}{R_{1}^{2}-r_{1}^{2}} \frac{1}{\rho}-(1-2 \mu) \frac{R_{1}^{2} q_{1}}{R_{1}^{2}-r_{1}^{2}} \rho\right]$

The radial stress is obtained as $13.89 \mathrm{MPa}$, and the hoop stress is 264.16MPa.

For steel sleeve, the stress field is:

$\sigma_{\rho}=-\frac{\frac{R_{2}^{2}}{\rho^{2}}-1}{\frac{R_{2}^{2}}{r_{2}^{2}}-1} q_{1} \quad \sigma_{\varphi}=\frac{\frac{R_{2}^{2}}{\rho^{2}}+1}{\frac{R_{2}^{2}}{r_{2}^{2}}-1} q_{1}$ 
$u_{\rho}=\frac{1+\mu}{E}\left[\frac{r_{2}^{2} R_{2}^{2} q_{1}}{R_{2}^{2}-r_{2}^{2}} \frac{1}{\rho}+(1-2 \mu) \frac{r_{2}^{2} q_{1}}{R_{2}^{2}-r_{2}^{2}} \rho\right]$

The radial stress is obtained as $13.89 \mathrm{MPa}$, and the hoop stress is $-125.64 \mathrm{MPa}$.

According to the settings in Section 2, a FEA model of steel sleeve joint is created in ABAQUS to simulate the interference fit assembly process specified in this section. The Mises stress contour of joint after interference fit is shown in Fig.9. Most regions of the components are in elastic stage. Fig.10, 11 show the comparison of both radial and hoop stresses from FEA model and theoretical analysis along the height of the column.

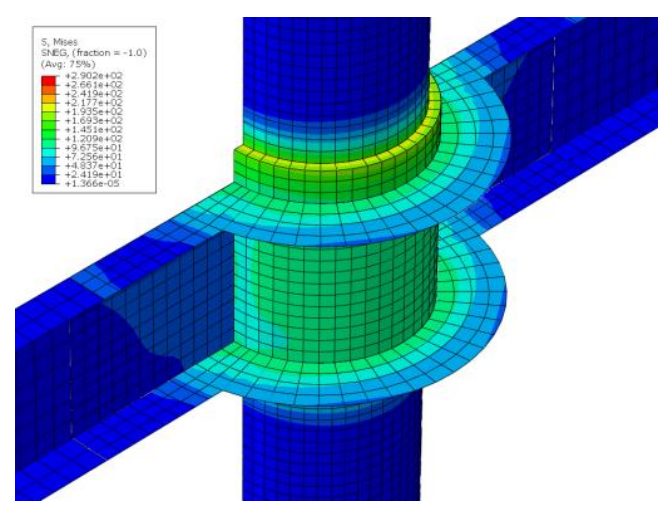

Fig. 3 Mises stress contour of joint after interference fit

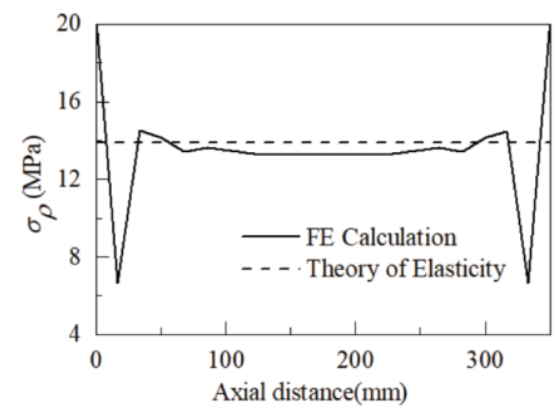

(a) Steel column

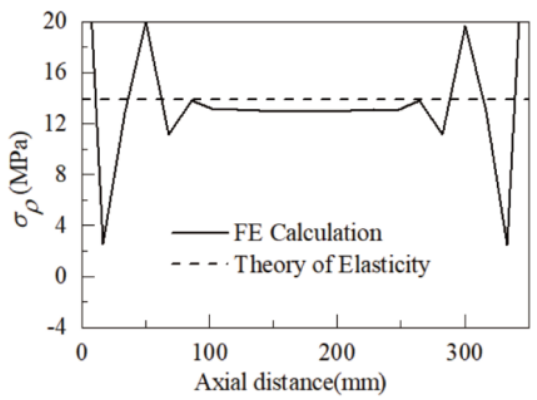

(b) Sleeve

Fig. 4 Comparison of $\sigma_{\rho}$ between elastic analysis and FE calculation

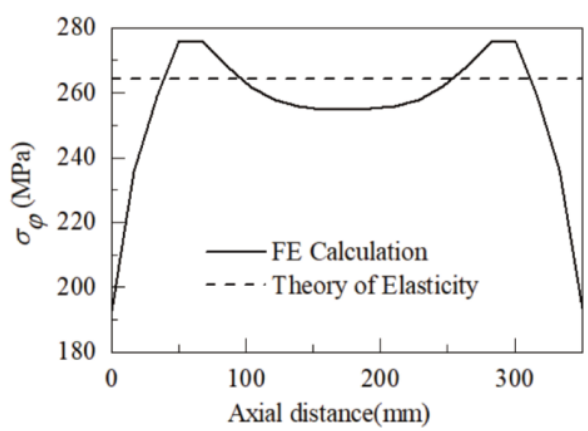

(a) Steel column

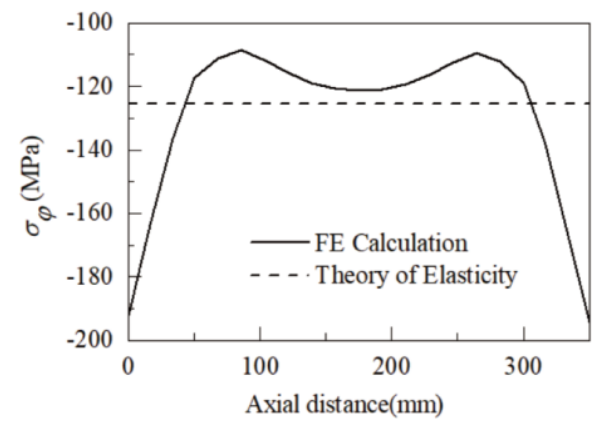

(b) Sleeve

Fig. 5 Comparison of $\sigma_{\varphi}$ between elastic analysis and FE calculation

It is seen from Fig.10 and 11 that the FEA results at both ends are different from the theoretical results due to the stress concentration. The stresses at height $50 \mathrm{~mm}$ and $300 \mathrm{~mm}$ are also different from the theoretical results due to the diaphragms. For the rest portion of the stress curves, the results of FEA and theoretical are in fairly good agreement..

The comparative studies in Section 3.1, 3.2 and 3.3 show that FEA model in ABAQUS matches closely with experimental study or theoretical calculation in terms of beam-column joint, CFST column as well as the interference fit. Therefore, the FEA model of SSBCJ-IF which is composed of the aforementioned three cases is highly reliable.

\section{Parametric analysis of moment-rotation (M- $\theta)$ relationship}

The parametric analyses of the SSBCJ-IF are performed from three aspects, material, geometry and load. The changing trend of the initial rigidity and capacity of the joint under each parameter is obtained. Also, a moment-rotation calculation model is established by using the results obtained from the parametric analyses. The location of the measuring point is shown in Fig.12.

\section{1. $M$ - $\theta$ relationship}
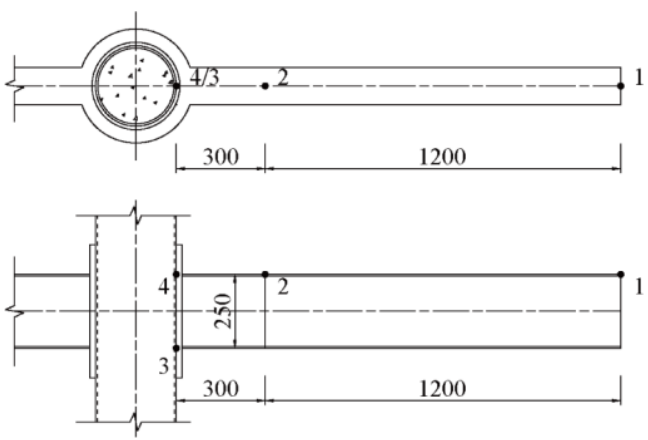

Fig. 6 Location of the measuring-point (mm)

The $M$ - $\theta$ relationship of the joint is calculated by Eq. (5) and (6), where $M$ is the moment and $\theta$ is the rotation of the joint [24].

$M=P L_{\text {load }}$ 
$\theta=\theta_{b}-\theta_{c}$

Where, $P$ is the load $(\mathrm{kN}) ; L_{\text {load }}$ is the distance from the location of the loading to the edge of the column which equals to $1.5 \mathrm{~m} ; \theta_{b}$ is the rotation angles of the beam while $\theta_{c}$ is the rotation angles of the column (rad).

The beam rotation $\theta_{b}$ is given by Eq. (7) and (8):

$\theta_{b}=\arctan \frac{\left|\delta_{\mathrm{DV} 1}-\delta_{\mathrm{bel}, \mathrm{DV} 1}\right|-\left|\delta_{\mathrm{DV} 2}-\delta_{\mathrm{bel}, \mathrm{DV} 2}\right|}{1200}$

$\delta_{\mathrm{bel}, \mathrm{DV} i}=-\frac{P}{E_{b} I_{b}}\left(\frac{x_{\mathrm{DV} i}^{3}}{6}-\frac{L_{\text {load }} x_{\mathrm{DV} i}^{2}}{2}\right)$

Where, $\delta_{\mathrm{DV} i}$ is the vertical displacement at measuring point $i(\mathrm{~mm})$; $\delta_{\text {bel,DV } i}$ is the calculated elastic displacement of the beam at DV $i(\mathrm{~mm}) ; E_{b}$ is the Young's modulus of the beam $(\mathrm{MPa}) ; I_{b}$ is the inertia moment of the steel beam section $\left(\mathrm{mm}^{4}\right) ; x_{\mathrm{DV} i}$ is the distance from the measuring point $i$ to the face of the column ( $\mathrm{mm})$.

The column rotation $\theta_{c}$ is given by Eq. (9):

$\theta_{c}=\arctan \frac{\left|\delta_{\mathrm{DH} 3}\right|+\left|\delta_{\mathrm{DH} 4}\right|}{250}$

Where, $\delta_{\mathrm{DHi}}$ is the horizontal displacement of the measuring point $i(\mathrm{~mm})$.

The initial stiffness of $K_{i}$ is taken as the secant rigidity corresponding to $0.2 M_{u}[1]$

$K_{i}=\frac{0.2 M_{u}}{\theta_{0.2}}$

Where, $\theta_{0.2}$ is the rotation angle corresponding to $0.2 M_{u}(\mathrm{rad}) ; M_{u}$ is the ultimate flexural strength of the joint $(\mathrm{kN} \cdot \mathrm{m})$

\subsection{Parametric studies}

The basic configuration of the joint is set as follows: The section of the steel tube column is $273 \times 7$ (diameter $\times$ wall thickness in $\mathrm{mm}$ ) filled with C40 concrete. The beam section height, width, web thickness and flange thickness is $250 \mathrm{~mm}, 125 \mathrm{~mm}, 6 \mathrm{~mm}$ and $9 \mathrm{~mm}$, respectively. The thickness of the external diaphragm is $9 \mathrm{~mm}$. The steel sleeve thickness is $16 \mathrm{~mm}$. The sleeve height are $350 \mathrm{~mm}$. The magnitude of interference is $0.5 \mathrm{~mm}$. The axial compression ratio is 0.3 . With this configuration, the parametric studies are performed for material parameter, geometric parameter as well as load parameter. Table 1 shows the values of parameter.

Table 1

Parameter detail Parameters

\begin{tabular}{|c|c|c|c|c|c|c|}
\hline \multicolumn{2}{|c|}{ Material } & \multicolumn{4}{|c|}{ Geometry } & \multirow{2}{*}{$\begin{array}{c}\text { Load } \\
n\end{array}$} \\
\hline$C$ & $Q$ & $\begin{array}{c}\delta \\
/ \mathrm{mm}\end{array}$ & $\alpha$ & $k$ & $k_{m}$ & \\
\hline $\mathrm{C} 40$ & Q235 & 0.1 & 0.077 & 0.257 & 0.418 & 0.1 \\
\hline C50 & Q345 & 0.3 & 0.111 & 0.300 & 0.606 & 0.3 \\
\hline C60 & Q390 & 0.5 & 0.164 & 0.375 & 0.764 & 0.5 \\
\hline C70 & Q420 & 0.7 & 0.202 & 0.449 & - & 0.7 \\
\hline $\mathrm{C} 80$ & Q460 & 0.9 & 0.242 & 0.529 & - & 0.9 \\
\hline- & - & - & 0.283 & 0.599 & - & - \\
\hline- & - & - & - & 0.691 & - & - \\
\hline
\end{tabular}

Note: $C$ - concrete grade; $Q$ - steel grade; $\delta$ - magnitude of interference; $\alpha$ - steel ratio of the column section; $k$ - beam-column linear stiffness ratio; $k_{m}$ - beam-column strength ratio; $n$ - axial compression ratio of the column.
(1) Concrete grade

Fig.13(a) shows that when concrete grade is within the range of $\mathrm{C} 40$ to $\mathrm{C} 80$ the flexural bearing capacity remains almost the same. This is because the failure mainly occurs at the beam flange and web near the column face while only small portion of the column section fails. The failure pattern of the joint satisfies the design principle of "strong column weak beam". Fig.13(b) shows that the initial rigidity increases slightly with the increase of the concrete grade This is because there is an interrelation between concrete grade and concrete elastic modulus. Higher concrete grade usually corresponds to higher elastic modulus.

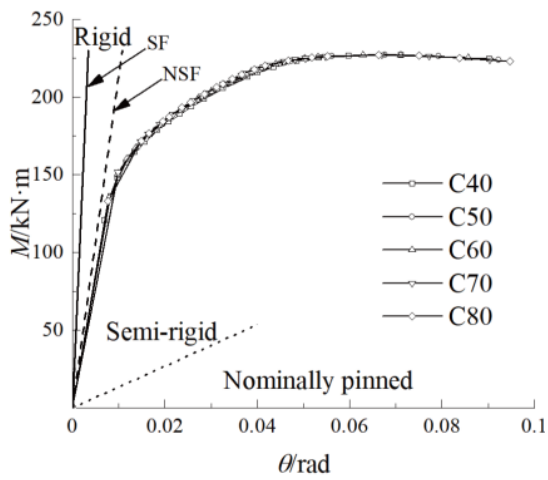

(a) $M-\theta$ relations

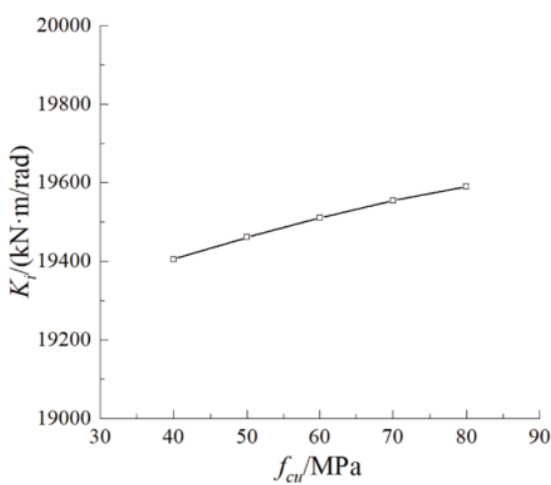

(b) Initial stiffness

Fig. 7 Influence of concrete strength

(2) Steel grade

Fig.14(a) shows that the flexural bearing capacity increases with the increase of the steel grade while the shape of the moment-rotation curves is similar with each other. The similar shape of the $M-\theta$ curves indicates the failure process is also similar. Fig.14(b) shows that the steel grade has without influence on the initial rigidity. This is because the elastic modulus of steel is not directly related to the steel grade.

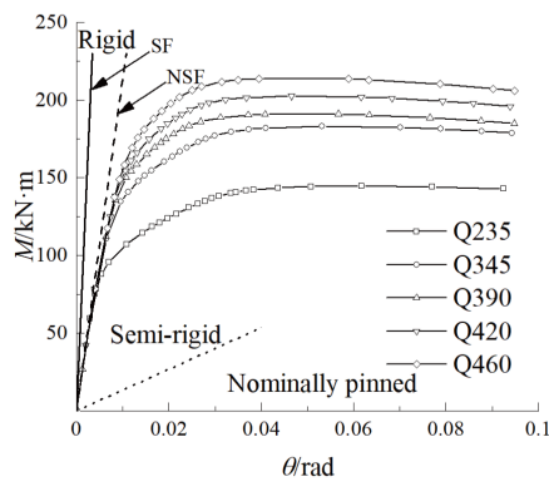

(a) $M-\theta$ relations 


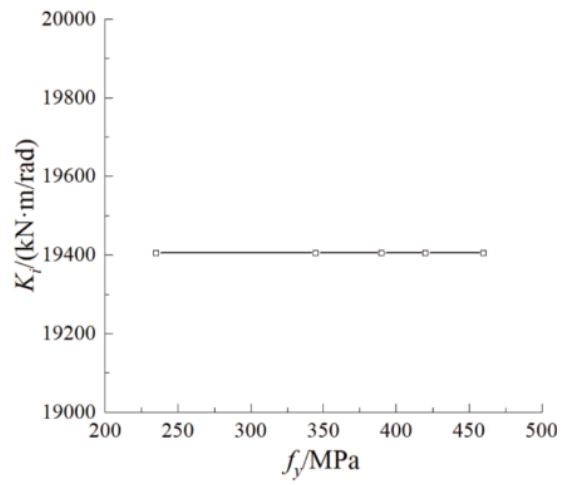

(b) Initial stiffness

Fig. 8 Influence of steel strength.

(3) Magnitude of interference

Fig. 15 shows that the flexural bearing capacity and the initial rigidity of joints increase with the increase of interference when the interference is less than 0.5 and decreases with the decrease of interference when the interference is more than 0.5 . When the interference is low, the pre-tightening force between the steel tube and sleeve is small. In this case, the pre-tightening force will be lost under relatively small external load resulting in the stiffness decrease. When the magnitude of interference is high, the pre-tightening force between the steel tube and sleeve is large. In this case, the sleeve reaches plastic stage due to the excessive stress introduced by the large pre-tightening force resulting in the stiffness decrease.

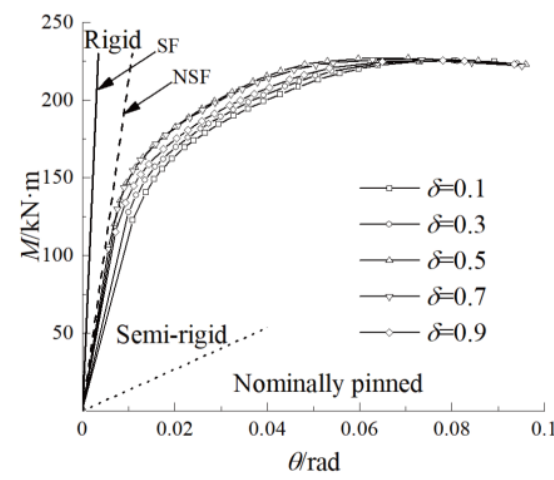

(a) $M-\theta$ relations

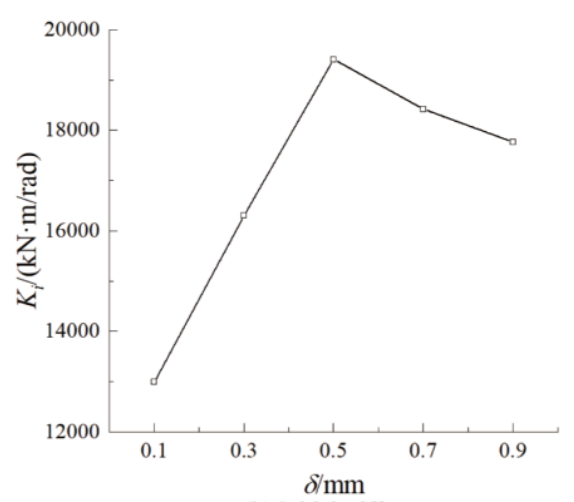

(b) Initial stiffness

Fig. 9 Influence of magnitude of interference

(4) Steel ratio

The steel ratio $\alpha=A_{s} / A_{c}$, where $A_{\mathrm{s}}$ is the areas of steel tube, $A_{\mathrm{c}}$ is the areas of core concrete. Fig. 16 shows that the initial rigidity and flexural capacity can be increased along with the increase of the steel ratio of column section. For specific performance: With the increase of the steel ratio, the restraint effect on the concrete is enhanced so that the bending rigidity of the column section is improved. Fig.16 shows that the steel ratio has a great influence on the initial rigidity. Because the major failure occurs at the beam sections near column face, the steel ratio only has relatively small influence on the bending capacity.

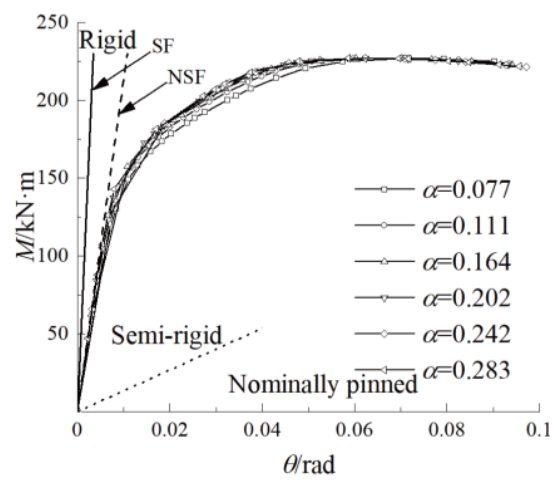

(a) $M-\theta$ relations

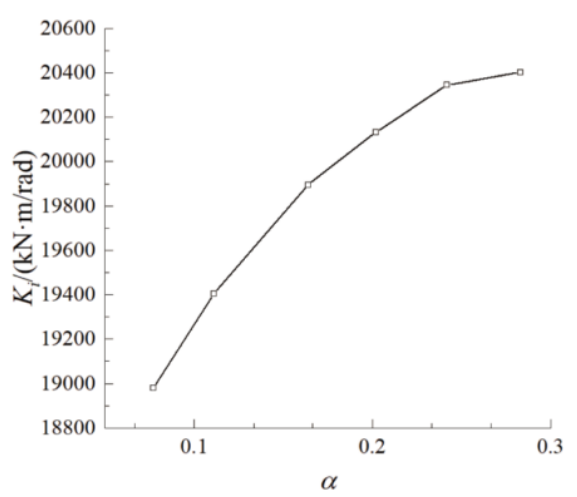

(b) Initial stiffness

Fig. 10 Influence of steel ratio

(5) Beam-column linear stiffness ratio

The beam-column linear stiffness ratio is defined as:

$$
k=\frac{(E I)_{b} H}{(E I)_{c} L}
$$

where $(E I)_{b}$ is flexural rigidity of beam, $(E I)_{c}$ is flexural rigidity of CFST column, $H$ is column height and $L$ is beam span length.

The variation in beam-column linear stiffness ratio ranging from 0.257 to 0.691 is realized by changing the beam span length. Fig.17 shows that the variation of linear stiffness ratio has little influence on the capacity, but it has a significant influence on the initial stiffness. The maximum initial stiffness is reached when the beam-column linear stiffness ratio is about 0.5 . To achieve the design goal and to improve the flexural rigidity of the joint, the linear stiffness ratio should not be too large. Thus, the optimum value of $k$ is about 0.5 .

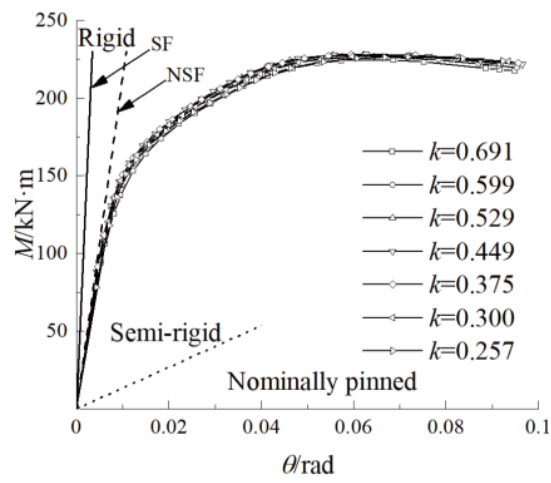

(a) $M-\theta$ relations 


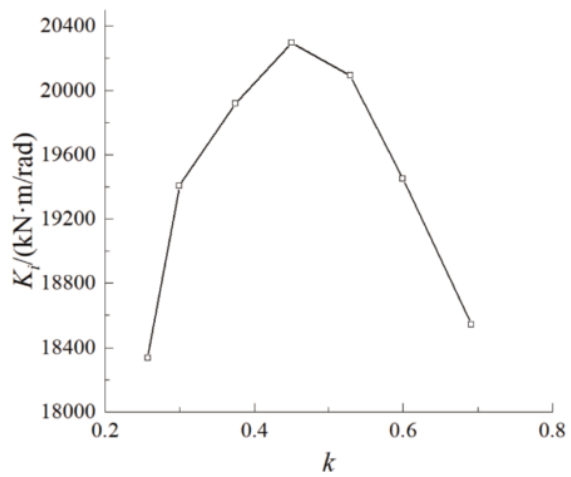

(b) Initial stiffness
(6) Beam-column strength ratio

The beam-column strength ratio is taken as $k_{m}=M_{u b} / M_{u c}$, where $M_{u b}, M_{u c}$ is the bending strength of the steel beams and column, respectively. Fig.18(a) shows that the ratio $k_{\mathrm{m}}$ has a great effect on the $M-\theta$ curve. This is because the variation of $k_{\mathrm{m}}$ is realized by adjusting the beam section and the beam section with higher bending strength will give both a higher flexural bearing capacity and a higher initial stiffness.

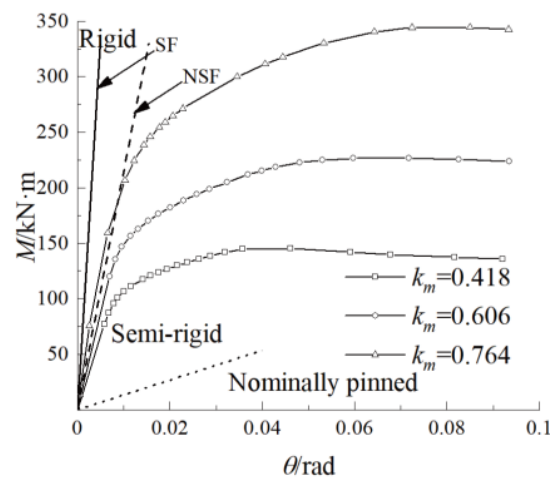

(a) $M-\theta$ relations

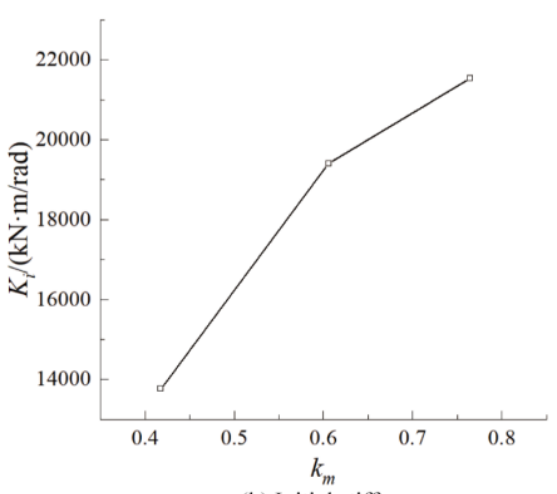

(b) Initial stiffness

Fig. 12 Influence of strength ratio of beam to column

(7) Axial compression ratio of the column

Fig.19 shows the relationship between axial load ratio and moment-rotation curve. The axial load ratio ranges from 0.1 to 0.9 . Both the flexural bearing capacity and initial rigidity of the joint remain the same when the axial compression ratio of column is between 0.1 and 0.7 . They both decrease when the axial compression load ratio is above 0.7 . This is because the large axial load ratio changes the failure pattern of the joint from beam failure to column compression-bending failure. This indicates that the failure of column prior to the beam failure should be avoided.

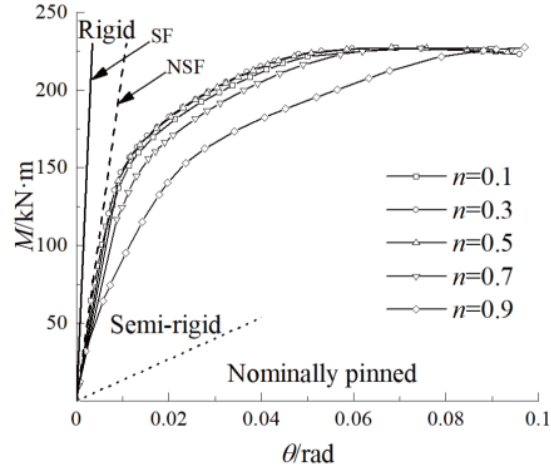

(a) $M-\theta$ relations

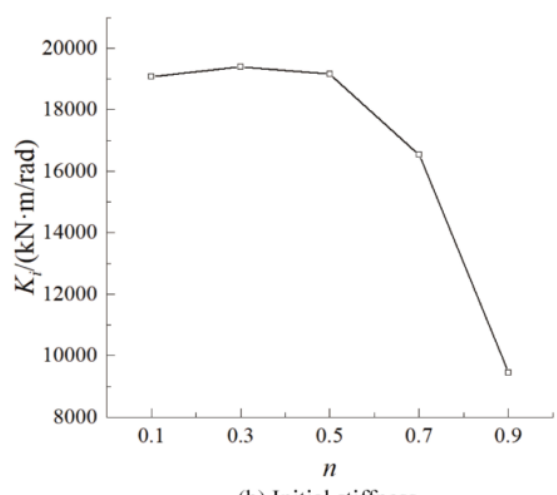

(b) Initial stiffness

Fig. 19 Influence of axial compression ratio

\subsection{Classification of joints}

Using the classification method in European Standard (EC3-2005) [25], the joints are divided into three types based on the initial rotational rigidity. If the initial rigidity $S_{j, \text { ini }} \geqslant k_{b} E I_{b} / L_{b}$, the joint is rigid. If $S_{j, \text { ini }} \geqslant 0.5 E I_{b} / L_{b}$, the joint is a nominally pinned joint. If $S_{j, \text { ini }}$ is between these two limits, the joint is semi-rigid. According to Fig.13-19, the SSBCJ-IF should be classified as a semi-rigid joint.

\section{Simplified calculation of $\mathrm{M}-\theta$ relationship}

The three-parameter power model proposed by Kishi et al [14] is used to describe the moment-rotation relation of CFST beam-column joints.

$\theta=\frac{M}{K_{i}} \frac{1}{\left[1-\left(M / M_{u}\right)^{n}\right]^{1 / n}}$

Where $k_{i}$ is the initial rigidity $(\mathrm{kN} \cdot \mathrm{m} / \mathrm{rad}) ; M_{u}$ is the ultimate flexural capacity $(\mathrm{kN} \cdot \mathrm{m}) ; n$ is the parameter related that affects the moment-rotation curve.

The three parameters in Eq. $11, K_{i}, M_{u}$ and $n$, are determined with numerical analysis as follows.

Initial stiffness $k_{i}$

The parametric analyses indicate that the major factors affecting the initial rigidity are axial compression ratio $n$, steel ratio $\alpha$, beam-column strength ratio $k_{m}$, linear stiffness ratio $k$ and magnitude of interference $\delta$.

The interference directly affects the radial contact pressure between the steel tube and the sleeve. The steel tube and the sleeve connection rely on the friction caused by the contact pressure to transfer and resist the load. The contact pressure can be calculate using linear elasticity method but solving system of equations is required in this method which is too complex. Here the contact pressure is obtained with the equation from mechanics of materials which is common in mechanical engineering as follows.

$\delta=q d\left(\frac{C_{1}}{E_{1}}+\frac{C_{2}}{E_{2}}\right)$ 
Where, $q$ is the pressure on the interference-fitted surface (MPa); $\mathrm{d}$ is the diameter of the interference-fitted surface $(\mathrm{mm}) ; E_{l}$ and $E_{2}$ are the Young's modulus of steel tube and sleeve, respectively $(\mathrm{MPa}) ; C_{l}$ is the stiffness coefficient of the steel tube, $C_{l}=\left(d^{2}+d_{l}^{2}\right) /\left(d^{2}-d_{l}^{2}\right)-\mu_{l} ; d_{1}$ is the inner diameter of the steel tube; $\mu_{1}$ is the Poisson's ratio of the steel tube; $C_{2}$ is the stiffness coefficient of the steel sleeve, $C_{2}=\left(d_{2}{ }^{2}+d^{2}\right) /\left(d_{2}{ }^{2}-d^{2}\right)+\mu_{2} ; d_{2}$ is the outer diameter of the steel sleeve $(\mathrm{mm}) ; \mu_{2}$ is the Poisson's ratio of the steel sleeve.

Using Eq. (12), the magnitude of interference can be converted into the corresponding contact pressure giving $q=2.69 \sim 24.22 \mathrm{MPa}$. The ranges of other parameters are as follows: $n=0.1 \sim 0.9, \alpha=0.077 \sim 0.283, k_{m}=0.418 \sim 0.764$, $k=0.257 \sim 0.691$. Based on these parameters, the equation for initial stiffness $K_{i}$ is defined as follows.

$$
K_{i}=R \cdot f(n) \cdot f(\alpha) \cdot f\left(k_{m}\right) \cdot f(k) \cdot f(q)
$$

Where, $R$ is a coefficient. $f(n), f(\alpha), f\left(k_{m}\right), f(k)$ and $f(q)$ are the mathematical relations between $K_{i}$ and $n, a, k_{m}, k$ and $q$.

$$
\begin{aligned}
& f(n)=\left(-2.13 n^{3}+1.44 n^{2}-0.11 n+1\right) \times 0.4 \\
& f(\alpha)=1.34 \ln (3.12 \alpha)+4.8 \\
& f\left(k_{m}\right)=3.97 k_{m}{ }^{2}+2.25 k_{m}+13.04 \\
& f(k)=-2.15 k^{2}+2.05 k+0.32
\end{aligned}
$$

$$
f(q)=\left(-q^{2}+37.18 q+432.11\right) \times 1.55
$$

Substituting Eq. (14) (18) into Eq. (13), the initial stiffness of the joint $K_{i}$ is given by:

$$
\begin{aligned}
K_{i}= & 0.62\left(-2.13 n^{3}+1.44 n^{2}-0.11 n+1\right) \\
& (1.34 \ln (3.12 \alpha)+4.8)\left(3.97 k_{m}{ }^{2}+2.25 k_{m}+13.04\right) \\
& \left(-2.15 k^{2}+2.05 k+0.32\right)\left(-q^{2}+37.18 q+432.11\right)
\end{aligned}
$$

Fig.20 shows the comparison between the initial stiffness from the proposed simplified calculation and that from the FEA modeling. It is seen that the two results are in good agreement with each other and the maximum error is less than $10 \%$

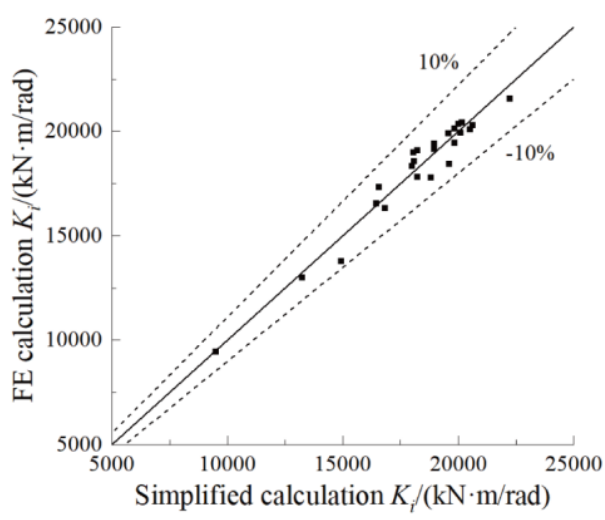

Fig. 13 Simplified calculation of initial stiffness compared with FEA calculation

Ultimate flexural capacity $M_{u}$ :

Since the failure is ensured to occur at the beam section near column face, the ultimate flexural bearing capacity of the joint $M_{u}$ is taken as the ultimate flexural bearing capacity of the beam.

$M_{u}=W_{n x} f_{u}$
Where, $W_{n x}$ is the net cross-section flexural modulus of the beam $\left(\mathrm{mm}^{3}\right) . f_{u}$ is the tensile strength of the beam (MPa).

Parameter $n$ :

Substitute $K_{i}$ and $M_{u}$ into Eq. (11). After data fitting, the relation between $n$ and $\theta_{0}$ is given by:

$n=0.48 \log _{10} \theta_{0}+2.5$

Where $\theta_{0}$ is the reference rotation angle, $\theta_{0}=M_{u} / K_{i}$.

Fig. 21 shows the comparisons between the $M-\theta$ curves from Eq. (11) and the curves from the FEA analysis. It is seen from the Fig.21 that the simplified calculation method can conservatively capture the nonlinear structural behavior of the joint.

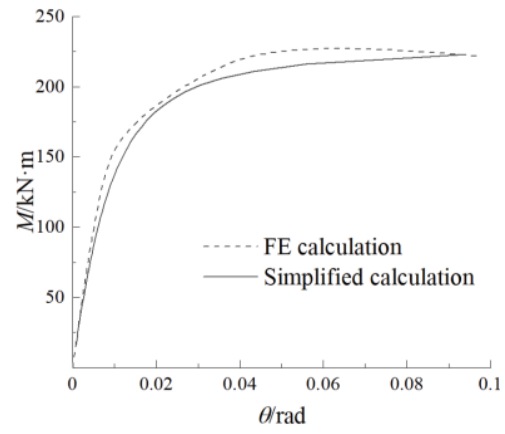

(a) $\alpha=0.202$

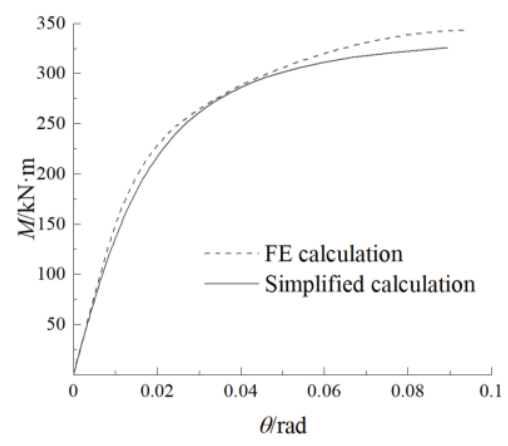

(b) $k_{m}=0.764$

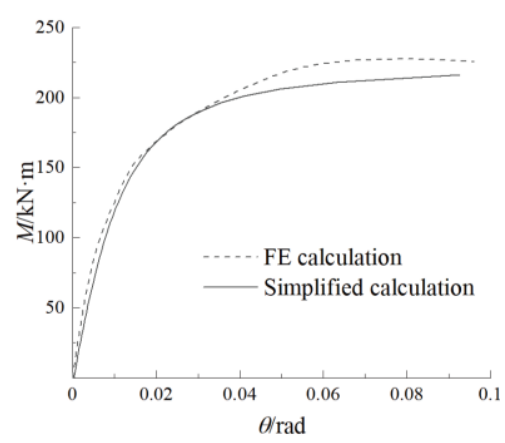

(c) $n=0.7$

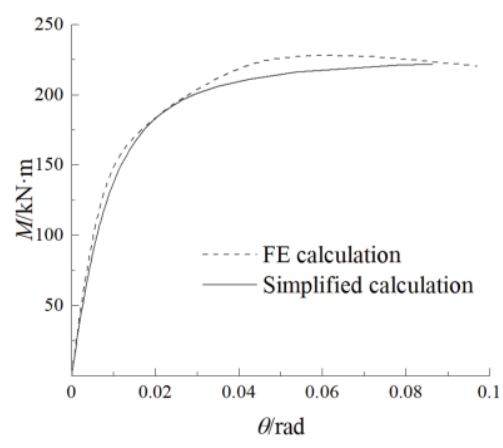

(d) $k=0.529$

Fig. 21 Moment-rotation curves (simplified calculation method vs FEA calculation) 


\section{Conclusions}

In this paper, the parametric analysis on the mechanical properties of SSBCJ-IF is performed. The influence of major parameters on the flexural bearing capacity and initial rigidity of the SSBCJ-IF are studied. One simplified calculation method for the moment-rotation curve is also proposed. The main conclusions are as follows.

(1) A FEA model of the SSBCJ-IF is proposed in this paper. The results from the FEA model are compared with the results from the experimental studies and theoretical calculation to verify the reliability of the FEA model.

(2) The parametric analysis is performed with three aspects, material, geometric and load. The results show that concrete strength increase had almost no influence on the flexural bearing capacity but has a small influence on the initial rigidity. The flexural capacity can be increases along with the increase of steel grade, and the steel grade has no effect on the initial stiffness. The stiffness of the joint reaches the peak when the magnitude of interference is $0.5 \mathrm{~mm}$ (corresponding to $13.45 \mathrm{MPa}$ of pre-tightening force). The magnitude of interference has little influence on the joint flexural capacity. Increasing the steel ratio can slightly increases the initial stiffness of the joint. To satisfy the design goal of "strong column weak beam", the beam-column linear stiffness ratio should not be too large and the joint can reach the maximum stiffness when the linear stiffness ratio is about 0.5. Increasing the beam-column strength ratio through adjusting beam section can improve the initial stiffness and flexural bearing capacity of the joint significantly. When the axial load ratio is 0.7 or less, the change of column axial force has little influence on the joint. When the axial load ratio exceeds 0.7 , the initial rigidity and the flexural bearing capacity both decrease significantly with the increase of the axial load ratio.

(3) The SSBCJ-IF is semi-rigid joint according to the classification method of European Standard (EC3) and it is similar with the rigid joint of braced frames.

(4) According to the results of the parametric analysis, a simplified calculation method for the $M-\theta$ relation is proposed by fitting the three-parameter power model. The $M-\theta$ curves obtained by the simplified calculation method are compared with the curves from the FEA method and the results match with each other very well.

\section{Acknowledgement:}

This research was funded by Science and Technology Research Key Project in Higher Institutions of Hebei Province (ZD2018250), Local Science and Technology Development Fund Project Guided by Central Government (206Z7601G) and Natural Science Foundation of Hebei Province (E2020210074).

\section{References:}

[1] Lin-Hai Han, Wei Li. Seismic performance of CFST column to steel beam joint with RC slab: Experiments[J]. Journal of Constructional Steel Research, 2010, 66(11): 1374-1386.

[2] Xi-Lin Liu, Yong Yu, Tanaka Kiyoshi. Experimental study on the seismic behavior in the connection between CFRT column and steel beam[J]. Structural Engineering and Mechanics, 2000, 9(4): 365-374.

[3] Jian-Guo Nie, Kai Qin, Rong Liu. Experimental study on seismic behavior of connections composed of concrete-filled square steel tubular columns and steel-concrete composite beams with interior diaphragms[J]. Journal of building structures, 2006, 27(4): 1-9.

[4] Ning Wang, Myung-Jae Lee. Structural behavior of beam-to-column connections of circular CFST columns by using mixed diaphragms[J]. International Journal of Steel Structures, 2015, 15(2): 347-364

[5] Rui Li, Bijan Sanali, Zhong Tao, Md Kamrul Hassan. Cyclic behaviour of composite joints with reduced beam sections[J]. Engineering Structures, 2017, 136: 329-344.

[6] Yan-Xia Zhang, Quan-Gang Li, Wei-Zhen Huang, Kun Jiang, Yu Sun. Behavior of prefabricated beam-column connection with short strands in self-centering steel frame[J]. Advanced Steel Construction, 2019, 15(2): 203-214.

[7] Atorod Azizinamini, Stephen P. Schneider. Moment connections to circular concrete-filled steel tube columns[J]. Journal of structural Engineering, 2004, 130(2): 213-222.

[8] Kyung-Jae Shin, Young-Ju Kim, Young-Suk Oh, Tae-Sup Moon. Behavior of welded CFT column to H-beam connections with external stiffeners[J]. Engineering Structures, 2004, 26(13): 1877-1887.

[9] Shen Yan, Kim J.R. Rasmussen, Lu-Li Jiang, Chen Zhu, Hao Zhang. Experimental evaluation of the full-range behaviour of steel beam-to-column connections[J]. Advanced Steel Construction, 2020, 16(1): 77-84.

[10] Shan Gao, Man Xu, Lan-Hui Guo, Su-Mei Zhang. Behavior of CFST-column to steel-beam joints in the scenario of column loss. Advanced Steel Construction, 2019, 15(1): 47-54.

[11] Lightfoot E, Messuries A P L. Elastic analysis of frameworks with elastic connections. Journal of the Structural Division, 1974, 100(6): 1297-1309.

[12] Frye M.John, Morris Glenn. Analysis of flexibly connected steel frames[J]. Canadian journal of civil engineering, 1975, 2(3): 280-291.

[13] Jones Stephen W, Kirby Patrick A, Nethercot David A. Columns with semirigid joints[J]. Journal of the Structural Division, 1982, 108(2): 361-372.

[14] N. Kishi, Wai-Fah Chen. Moment-rotation relations of semirigid connections with angles[J]. Journal of Structural Engineering, 1990, 116(7): 1813-1834.
[15] Yee Yoke Leong, Melchers Robert E. Moment-rotation curves for bolted connections[J]. Journal of Structural Engineering, 1986, 112(3): 615-635.

[16] Gang Shi, Xue-Sen Chen. Moment-rotation curves of ultra-large capacity end-plate joints based on component method[J]. Journal of Constructional Steel Research, 2017, 128 : 451-461.

[17] G.C. Li, C.Y. Di, L. Tian, C. Fang. Nonlinear finite element analysis on long columuns of high-strength concrete-filled square steel tube with inner cfrq circular tube under axial load[J]. Advanced Steel Construction, 2013, 9(2): 124-138.

[18] Yong-Jiu Shi, Meng wang, Yuan-Qing Wang. Study on constitutive model of structural steel under cyclic loading[J]. Engineering Mechanics, 2012, 29(9): 92-98+105.

[19] GB/T 50081-2002 Chinese standard for test method of mechanical properties on ordinary concrete. Beijing: China Construction Industry Press,2002

[20] Lin-Hai Han, Guo-Huang Yao, Zhong Tao. Performance of concrete-filled thin-walled stee tubes under pure torsion[J]. Thin-Walled Structures, 2007, 45(1): 24-36.

[21] CORP D S S. ABAQU 6.14 Analysis user's guide volume V: Prescribed Conditions, Constraints \& Interactions [M]. USA: ABAQUS, Inc, Dassault Systemes, 2014.

[22] Ming-Lie Yan, Jun-Ping Wang, Zhang-Tao Li, Si-Mao Ren. Experimental study on sleeve joints of round steel tubes[J]. Building Structure, 2019, 49(06): 66-68.

[23] Lin-Hai Han, You-Fu Yang. Cyclic performance of concrete-filled steel CHS columns under flexural loading[J]. Journal of Constructional Steel Research, 2005, 61(4): 423-452.

[24] Xu-Hong Qiang, Nian-Du Wu, Yong-Feng Luo, Xiao Liu, Xu Jiang. Performance test and Finite element Analysis of overhanging End-plate joints in high strength steel[J]. Journal of Hunan University (Natural Science edition), 2018, 45(07): 1-9.

[25] CEN E. Eurocode 3: Design of steel structures Part 1-8: Design of joints[S]. 2005. 